\title{
A Preliminary Analysis of Wireless Link for Bats Signals Acquisition System
}

\author{
K. Herman ${ }^{a, *}$, M. Debski $^{a}$ And J. Furmankiewicz ${ }^{b}$ \\ ${ }^{a}$ Wrocław University of Technology, Wybrzeże Wyspiańskiego 27, 50-370 Wrocław, Poland \\ ${ }^{b}$ University of Wrocław, Pl. Uniwersytecki 1, 50-137 Wrocław, Poland
}

\begin{abstract}
The scope of an article that is presented below covers a preliminary analysis of a wireless link of ultrasound signal transmission in commercial radio frequency band (UHF). The radio link is used to transmit short ultrasound bat-like biosonar signals. Because of complicated structure properties and of transmitted signals the various methods of wireless transmission are shown. The hardware implementation consists of micro-electro-mechanical system microphone, microphone preamplifier and FM transmitter. At the end of the radio link a standard FM receiver is used. Since we use frequency modulation an influence of the radio link on received signal parameters is presented. The analysis of results provides us a set of information that might be used in designing of large scale wireless sensor networks for bats behavior monitoring.
\end{abstract}

PACS: 47.63.Cb, 87.19.U-, 07.05.Tp

\section{Introduction}

One of the basic methods of monitoring the activity of bats involves the use of ultrasonic detectors. Detection and acquisition of acoustic echolocation and social signals makes it possible for experienced chiropterologists to identify individual features of these animals (e.g. species). The properties of particular detection systems and their effect on the quality of the tested signal have been covered by a number of works [1-5]. It is, however, notable that all such devices have to be controlled by an operator. Increasing importance of environmental protection encourages researchers, who work to provide a broader perspective on the topic of spatial distribution of bats within a given area, which includes migration of those mammals [3]. The research methods used by chiropterology experts require a large measurement area to be monitored. As it was necessary to synchronise events in numerous points in space and centralise data acquisition, scientists began working on the construction of a dedicated wireless system for acquisition of bats' signals.

\section{The analysis of the possibilities of tele-transmission of bats' signals}

Previous environment monitoring methods were based on ultrasonic detectors converting ultrasound signals to frequencies audible to human ear. The transformed signals were saved by the detector using commercially available external recorder or by a built-in one. Table shows

\footnotetext{
* corresponding author; e-mail: Krzysztof .Herman@pwr.wroc.pl
}

a comparison of detection methods, which takes into account their effect on signal analysis. The following symbols were used to represent detection systems: ZC zero crossing, HET - heterodyne, DIV — divider, TE — time expansion, HF — high frequency.

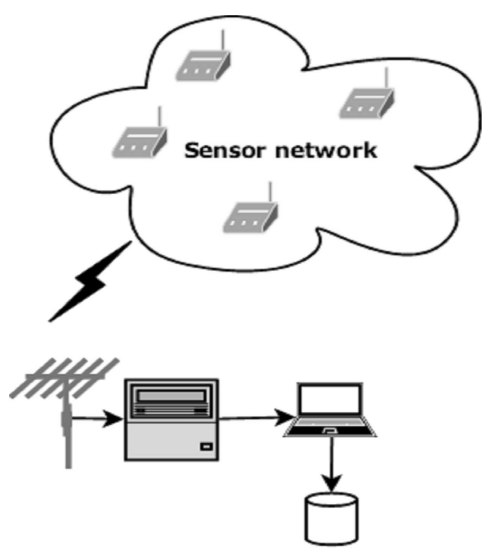

Fig. 1. Diagram representation of the multipointpoint transmission system.

Table shows that the HF method provides the most detailed set of information on a signal. If it is assumed that the upper threshold of the recorded signal is $150 \mathrm{kHz}$ and the dynamics about $60 \mathrm{~dB}$, it is possible to calculate the requirements concerning signal registration/transmission using the theory of analogue-digital processing. Unfortunately, HF registration also requires very high capacity connection, which becomes a serious obstacle when using numerous channels. The available wireless network 
protocols, which are based on packet transmission, do not guarantee the required and critical, from the environment monitoring point of view, accuracy in synchronising and defining individual events in time.

Comparison of bats' signals acquisition methods.

TABLE

\begin{tabular}{l|c|c|c|c|c}
\hline \hline & ZC & HET & DIV & TE & HF \\
\hline signal in time domain reproduction & good & good & good & very good & very good \\
envelope reproduction & unavailable & very bad & satisfactory & very good & very good \\
pulse sequence reproduction & good & good & good & unavailable & very good \\
signal spectrum components reproduction & satisfactory & unavailable & satisfactory & very good & very good \\
signal spectrogram reproduction & satisfactory & unavailable & satisfactory & very good & very good \\
required memory capacity for recording & $15 \mathrm{kbit} / \mathrm{s}$ & $120 \mathrm{kbit} / \mathrm{s}$ & $320 \mathrm{kbit} / \mathrm{s}$ & $320 \mathrm{kbit} / \mathrm{s}$ & $3.2 \mathrm{Mbit} / \mathrm{s}$
\end{tabular}

The aim of the system is to combine multipoint-point transmission with recording. Figure 1 shows the diagram representation of the above.

Individual sensors are managed from a base transceiver station (BTS) and are capable of simultaneous data transmission to the BTS, where the acquisition process takes place. Since unprocessed HF signal contains most information, attempts were made to set up transmission in a fashion that allows acquisition of such signal. It must also be noted that signal transmission has to cover a distance of several kilometres.

\section{Designing a wireless point-to-point connection system}

Most of the currently available wireless link solutions are systems based on digital modulations with packet data transmission. The available wireless network solutions are capable of transmitting data at a rate of $54 \mathrm{Mbit} / \mathrm{s}$. However, the range and time-lags occurring during transmission eliminate this type of connectivity from use in the studied case.

Analysis of wireless link in the context of designing a system revealed that it is possible to transmit signal in analogue form using analogue modulation.

The use of analogue signal transmission allows retaining time relations, which are a valuable source of information related to system operation. Initial research covered point-to-point signal transmission and testing the effect of transmission on the studied parameters of the transmitted signal. Analysis of analogue modulation methods and their capabilities related to transmission of such a broad band with signal noise-ratio ratio that allows correct detection of signal by a receiver resulted in selection of frequency modulation (FM). The key parameter in case of FM is frequency modulation index $m_{f}$ :

$$
m_{f}=\frac{\Delta f}{f_{m}},
$$

where $\Delta f$ - frequency deviation $[\mathrm{Hz}], f_{m}-$ maximum frequency of the modulating signal $[\mathrm{Hz}]$.
Block diagram of a device realising point-to-point transmission is shown in Fig. 2.

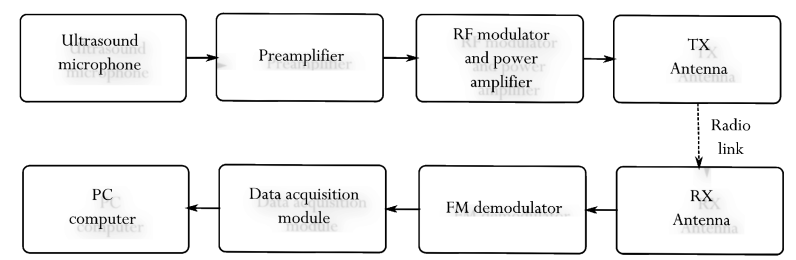

Fig. 2. Block diagram of point-to-point transmission.

The transmitting part consists of a micro-electro-mechanical system (MEMS) broadband ultrasonic microphone, a preamplifier and a FM modulator, which together with the amplifier emits electromagnetic wave via a TX antenna. The receiver consists of a receiving antenna, an FM demodulator with phase locked loop (PLL) and a signal acquisition system. Ultrasonic signal propagating in air is transformed to electric signal modulating $88 \mathrm{MHz}$ signal carrier. Signal reception, detection and acquisition is performed on the receiving end.

\section{Measurement results}

In order to show an influence of a radio transmission method on transmitted signal, the measurement system and the results were grouped in this section. The results were grouped and shown together with the measurement system. Analysis of the results covers time and frequency domain. In order to test system dynamics, measurements of transfer function in the setup were performed, as shown in Fig. 3.

The results of measurements for various transmitted frequencies are shown in Fig. 4.

Since output signal level depends on frequency, the research team measured frequency characteristics in the setup is shown in Fig. 5.

Figure 6 shows the results of the measurements.

As the next stage, the team performed measurements the aim of which was to test the accuracy of reproduction 


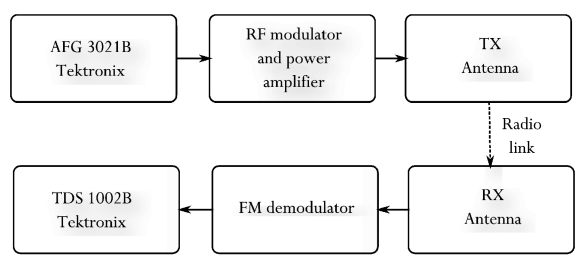

Fig. 3. Measurement diagram for measurement of the dynamics of the designed connection using an AFG 3012B generator and a Tektronix TDS 1002B oscilloscope.

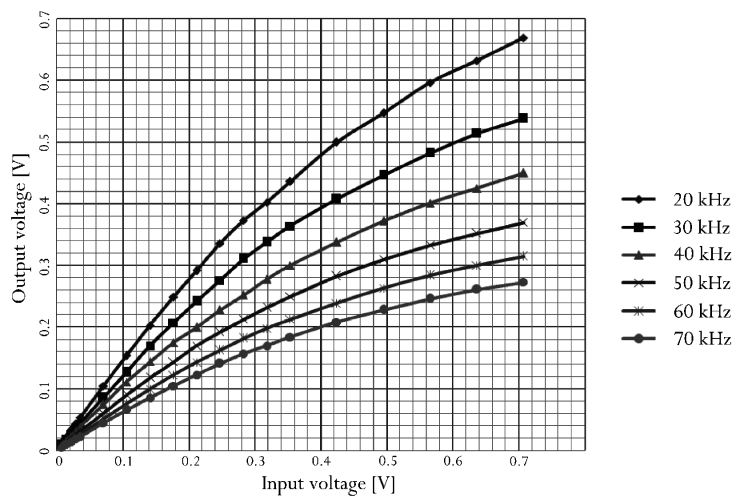

Fig. 4. Results of measurements of the signal dynamics of a point-to-point connection.

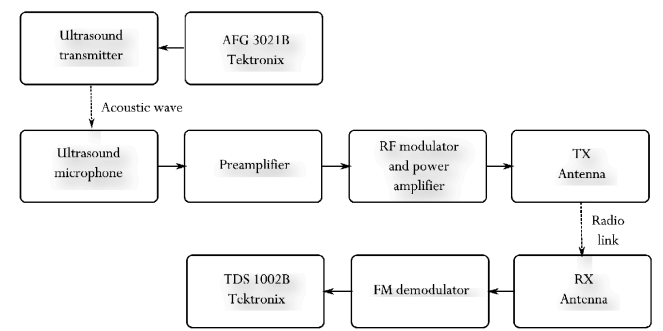

Fig. 5. Block diagram of a measurement setup with time analysis of signals.

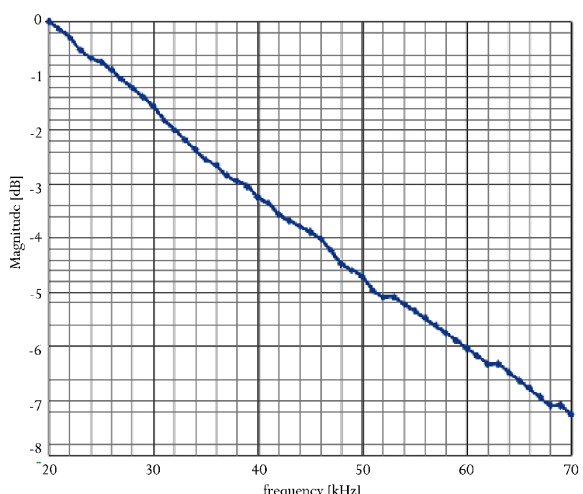

Fig. 6. Frequency characteristics of a point-to-point connection measured in the setup presented in Fig. 3. of pulse sequences and received signal spectrum with actuation by sinusoidal signal. Figures 7 and 8 show the results.
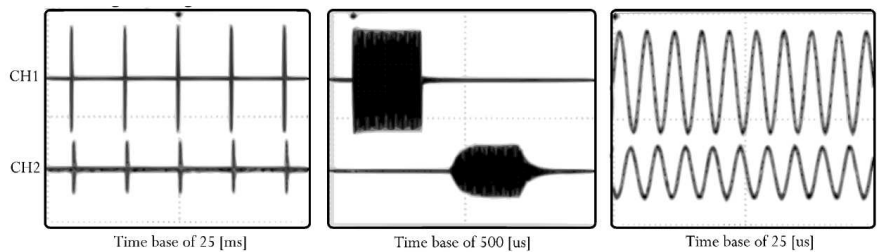

Fig. 7. The analysis of the accuracy of pulse reproduction performed on a setup presented in Fig. 5. $\mathrm{CH} 1-$ generator signal, $\mathrm{CH} 2$ - signal at the receiver's output.

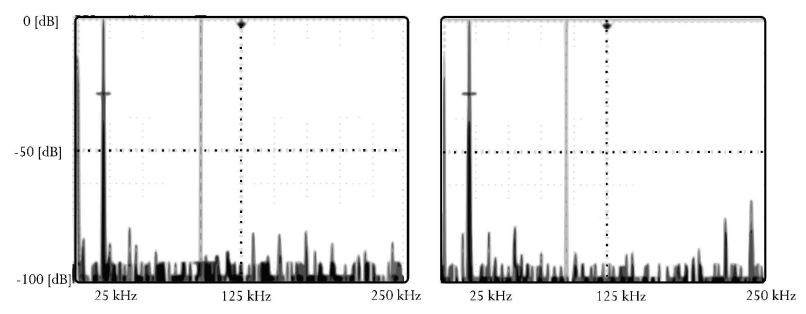

Fig. 8. Analysis in frequency domain. Transmitted signal (left), received signal (right).

\section{Conclusions}

The initial analyses of the possibilities of transmission of signals similar to those produced by bats can be a basis for developing the method used in the research. Analogue signal modulation and transmission guarantees acceptable signal parameters from the point of view of a biologist. Ultrasonic signals transmitted through the presented radio link are not significantly distorted and retain time relations. During the research a paper [6] was published, the authors of which used transmission based on analogue methods in laboratory tests related to bats' echolocation. This only reinforced the conviction that it was a right decision to use the selected method. The analyses and results related to technological possibilities of bat signals tele-transmission were presented in the above mentioned paper. Working in the frequency range covering radio band forced the authors to lower the emission power of the transmitter to $10 \mathrm{~mW}$. This limited the range of the device to several meters. The planned future work on developing the system will aim to design a multichannel link in ISM $866 \mathrm{MHz}$ band, which will use suitable channel separation and higher power and as a result will be able to perform multichannel transmission over significant distances. Over 10 time higher frequency of carrier wave will allow increased modulation index, which will improve signal to noise ratio. Construction of a dedicated environment monitoring system which utilises wireless connectivity also allows large-scale research and observation of bats. 


\section{References}

[1] I. Ahlen, H.J. Baagoe, Acta Chiropterologica 1, 137 (1999).

[2] M.B. Fenton, S. Bouchard, M.J. Vonhof, J. Zigouris, J. Mammalogy 82, 721 (2001).

[3] J. Furmankiewicz, M. Kucharska, J. Mammalogy 90, 1310 (2009).
[4] T. Gudra, K. Herman, Acta Bio-Optica Inform. Med. 15, 91 (2009).

[5] S. Hiryu, T. Hagino, H. Riquimaroux, Y. Watanabe, J. Acoust. Soc. Am. 121, 1749 (2007).

[6] S.M. Whiteley, D.A. Waters, G. Hayward, S.G. Pierce, I. Farr, Bioinspiration Biomimetics 5, 119 (2010). 